\title{
Sensory Experience Alters Specific Branches of Individual Corticocortical Axons during Development
}

\author{
Randy M. Bruno, ${ }^{1,2}$ Thomas T. G. Hahn, ${ }^{1,3}$ Damian J. Wallace, ${ }^{1,4}$ Christiaan P. J. de Kock, ${ }^{1,5}$ and Bert Sakmann ${ }^{1,6}$ \\ ${ }^{1}$ Department of Cell Physiology, Max Planck Institute for Medical Research, 69120 Heidelberg, Germany, ${ }^{2}$ Department of Neuroscience, Columbia \\ University, New York, New York 10032, ${ }^{3}$ Department of Psychiatry, Central Institute for Mental Health, 68072 Mannheim, Germany, ${ }^{4}$ Max Planck Institute \\ for Biological Cybernetics, 72076 Tübingen, Germany, ${ }^{5}$ Department of Integrative Neurophysiology, VU University Amsterdam, $1081 \mathrm{HV}$ Amsterdam, The \\ Netherlands, and ${ }^{6}$ Max Planck Institute of Neurobiology, 82152 Martinsried, Germany
}

Sensory experience can, over the course of days to weeks, produce long-lasting changes in brain function. Recent studies suggest that functional plasticity is mediated by alterations of the strengths of existing synapses or dynamics of dendritic spines. Alterations of cortical axons could also contribute to functional changes, but little is known about the effects of experience at the level of individual corticocortical axons. We reconstructed individual layer (L) 2/3 pyramidal neurons filled in vivo in developing barrel cortex of control and partially sensory-deprived rats. L2 axons had larger field spans than L3 axons but were otherwise equivalently affected by deprivation. Whisker trimming over $\sim 2$ weeks markedly reduced overall length of axonal branches in L2/3, but individual horizontal axons were as likely to innervate deprived areas as spared ones. The largest effect of deprivation was instead to reduce the length of those axonal branches in L2/3 oriented toward deprived regions. Thus, the location of a branch relative to its originating soma, rather than its own location within any specific cortical column, was the strongest determinant of axonal organization. Individual axons from L2/3 into L5/6 were similarly altered by whisker trimming although to a lesser extent. Thus, sensory experience over relatively short timescales may change the patterning of specific axonal branches within as well as between cortical columns during development.

\section{Introduction}

Sensory systems are topographically organized into maps, which are reorganized by experience (for review, see Buonomano and Merzenich, 1998; Calford, 2002; Fox, 2002; Kaas, 2002). The strength and short-term dynamics of existing synapses are experience-dependent (Finnerty et al., 1999), but synaptogenesis could also underlie map plasticity (Elston and DeFelipe, 2002; Kasai et al., 2003; Chklovskii et al., 2004; Bourne and Harris, 2007). Spines grow and retract after innocuous manipulations in adolescents and adults (Lendvai et al., 2000; Trachtenberg et al., 2002), but their extent is yet unclear (Xu et al., 2007). Larger structural modifications may transpire, but dendritic arbors are grossly reshaped only by destroying the periphery (Feldman and Brecht, 2005) and therefore an unlikely locus.

Large-scale axonal development and reorganization, obvious candidate mechanisms of map plasticity, have received less attention (Feldman and Brecht, 2005; Fox and Wong, 2005). Individual axons often have millimeter field spans but submicron diameters, complicating accurate three-dimensional reconstruction, and their substantial depth typically renders them inaccessible to in vivo imaging. Bulk tracing studies have provided evidence for

\footnotetext{
Received Dec. 12, 2008; revised Jan. 12, 2009; accepted Feb. 1, 2009.

This work was supported by the Max Planck Society. We thank Lisa Baumann, Sebastiano Bellanca, Björn Gärtner, Verena Hotz, Merle Kurz, Thorben Kurz, Ruta Masionyte, and Sara Tokur for reconstructions; Marlies Kaiser and Ellen Stier for histology; Philip Broser, Pavel Osten, and Verena Wimmer for help with trimming; and Michael Brecht for assistance in pilot work.

Correspondence should be addressed to Randy M. Bruno at the above address. E-mail: rb2604@columbia.edu. D0I:10.1523/JNEUROSCI.5911-08.2009

Copyright $\odot 2009$ Society for Neuroscience $\quad 0270-6474 / 09 / 293172-10 \$ 15.00 / 0$
}

malleability of cortical axons (Löwel and Singer, 1992; Trachtenberg and Stryker, 2001; Broser et al., 2008). Quantifying populations, however, is challenging and precludes measurement of actual axonal length. Furthermore, such studies cannot address questions at the level of individual axons.

Pioneering reconstructions of single geniculocortical axons bulk labeled in monocularly deprived cats showed that sensory biases correspondingly bias thalamocortical development (Antonini and Stryker, 1993a,b, 1996, 1998; Antonini et al., 1998). Single corticocortical axons have not been examined in this manner but are sensitive to global reductions in neural activity in vitro (Callaway and Katz, 1991; Dantzker and Callaway, 1998). The impact of biased sensory input on individual cortical axons has not been previously studied in vitro or in vivo.

Barrel cortex is ideal for investigating map plasticity. Facial whiskers of rodents are stereotypically arranged, corresponding one-toone to cortical barrels (Simons, 1978), anatomical landmarks facilitating interpretation of changes. A variety of whisker trimming protocols triggers functional plasticity, particularly in L2/3 (Fox, 2002; Feldman and Brecht, 2005; Fox and Wong, 2005). For example, whisker stimulation evokes voltage-sensitive dye signals that normally spread symmetrically into neighboring rows (Kleinfeld and Delaney, 1996; Sheth et al., 1998). After repeated trimming of rows $\mathrm{A}-\mathrm{C}$ from $\mathrm{P}$, stimulation of D-row whiskers evokes signals that spread preferentially toward the spared E row (Wallace and Sakmann, 2008). Bulk labeling reveals asymmetrical development of L2/3 axonal populations, a likely anatomical correlate of functional reorganization (Broser et al., 2008). These changes occur at ages when L2/3 axons are developing (Larsen and Callaway, 2006). 
To examine precisely how sensory experience alters development of individual corticocortical axons, we labeled single L2/3 pyramidal neurons in barrel cortex of normal and sensorydeprived rats. Surprisingly, trimming induced relatively little change of axonal length in spared and deprived columns. Deprivation instead primarily reduced, in both supragranular and infragranular layers, the arborization of those branches oriented toward deprived regions. Thus, sensory experience alters specific branches within as well as between cortical columns.

\section{Materials and Methods}

Whisker trimming. Eighty-three Wistar rats (Charles River) were used for these experiments. Whisker rows A, B, and C (including the straddlers $\alpha$, $\beta$, and $\gamma$ ) on the right side of the face were trimmed from postnatal day 7 . Whiskers were trimmed to a length of $<1 \mathrm{~mm}$ every day for $12-17 \mathrm{~d}$ before cell filling. Trimming was conducted without anesthesia. Deprived rats were housed in the same cages as control littermates, which were handled identically. The whiskers of control rats were sham trimmed by gentle brushing with scissors.

Surgery and cell filling. Rats were initially anesthetized with isoflurane and then transferred to urethane (1.6 g/kg, i.p.; $10 \%$ supplements given as necessary). Body temperature was maintained at $37^{\circ} \mathrm{C}$ by a servocontrolled heating blanket. The parietal and occipital bones were exposed, and a metal post or plate for positioning the head was attached to the skull using dental acrylic. In a few experiments the head was instead fixed in a stereotaxic frame. The skull overlying left barrel cortex was thinned with a dental drill until transparent.

To target neurons located in D row, all four experimenters used intrinsic signal imaging (Grinvald et al., 1986; de Kock et al., 2007). Hemodynamic responses to whisker deflection were imaged through the thinned bone. Individual whiskers or stumps were deflected several degrees by a piezo (5-10 deflections applied at 5-20 Hz). Responses were imaged (5-25 $\mathrm{Hz}$ frame rate) through a red bandpass or highpass filter and averaged over 30-160 trials (supplemental Fig. S1 B, available at www.jneurosci.org as supplemental material). The early (800-1200 ms after stimulus) intrinsic signal has a half-width corresponding to approximately the diameter of a single barrel (supplemental Fig. S1 B, available at www.jneurosci.org as supplemental material). The contours of the intrinsic signal were superimposed on an image of the vasculature acquired using a green filter, to determine the location of D row (supplemental Fig. S1C, available at www.jneurosci.org as supplemental material). Craniotomies (typically $<0.5 \mathrm{~mm}^{2}$ ) were then made above one or more D-row barrel columns.

Three of the experimenters occasionally used extracellular recording and manual whisker stimulation as a confirmation of, or an alternative to, intrinsic signal imaging. Extracellular recordings were made using glass pipettes that had tip inside diameters of $\sim 5 \mu \mathrm{m}$ and were filled with artificial CSF (aCSF) (in mm: $135 \mathrm{NaCl}, 5.4 \mathrm{KCl}, 1.8 \mathrm{CaCl}_{2}, 1.0 \mathrm{MgCl}_{2}$, and 5.0 HEPES, $\mathrm{pH}$ 7.2). Based on the success rate of recovering neurons in the appropriate location, the electrophysiological and imaging methods appeared equivalently reliable in targeting neurons located in D row.

Cells were filled either by whole-cell recording or juxtasomal filling. For whole-cell recording, patch pipettes were pulled from unfilamented borosilicate glass using a 3-or 4-stage pull. Inside tip diameters were $\sim 0.5 \mu \mathrm{m}$ wide. Pipettes were tip-filled with (in mM) $135 \mathrm{~K}$-gluconate, 10 HEPES, 10 phosphocreatine-Na, $4 \mathrm{KCl}, 4$ ATP-Mg, 0.3 guanosine triphosphate, and $0.2 \%$ biocytin ( $\mathrm{pH} 7.2$, osmolarity 291). Cells were searched for using current pulses (Margrie et al., 2002). Recordings were made in Bridge mode for typically 20-60 min. Similar pipettes, filled instead with aCSF containing $2 \%$ biocytin, were used for juxtasomal filling. Juxtasomal pipettes were inserted perpendicular to the pia. After a single-cell recording was established, square current pulses $(+1-7 \mathrm{nA}$, $200 \mathrm{~ms}$ on, $200 \mathrm{~ms}$ off) were passed for several minutes (Pinault, 1996). Regardless of the filling method, 1-2 h were usually allowed to elapse before perfusion to ensure adequate diffusion of the tracer. The two filling methods were equivalent in terms of labeling quality (see below).

To insure accurate axonal reconstruction, typically no further pipette penetrations were made after one neuron was thought to be successfully filled in an animal. A second attempt was occasionally made but in such cases would be placed 2-3 barrel columns away from the first.

Histology and reconstructions. The rat was deeply anesthetized and perfused transcardially with $0.1 \mathrm{~m}$ sodium phosphate buffer followed by $4 \%$ paraformaldehyde (in $0.1 \mathrm{~m}$ sodium phosphate buffer). Barrel cortex was cut tangentially in $100 \mu \mathrm{m}$ sections on a vibratome. Sections at the depth of layer 4 were stained for cytochrome oxidase (CO), and all sections were subsequently stained for biocytin (Horikawa and Armstrong, 1988). Cells were not recovered from every animal, likely because of a variety of complications: insufficient amount of filling, rupturing of the neuron during electrode withdrawal, respiratory problems preventing animal survival, inadequate perfusion, tissue damage during sectioning, or technical issues during staining.

Sixty-two cells were recovered in total. We reconstructed only those cells that had pyramidal cell bodies in L2/3, well filled apical dendrites, substantial numbers of visible spines, and a main axonal trunk passing through a D-row barrel. Superficial pyramidal neurons having apical dendrites oriented horizontally were included in this selection. Twentythree neurons met these criteria; the excluded cells were either poorly filled, L4 neurons, interneurons, or septum-related L2/3 neurons. Thirty-nine percent (14 of 36) of the whole-cell fills and 35\% ( 9 of 26) of the juxtasomal fills were deemed suitable for analysis, indicating that both techniques fill neurons equally well. All reconstructed intracellularly filled cells had action potential half-widths $>1 \mathrm{~ms}$ during recording, and all reconstructed juxtasomally filled cells had total waveform duration $>750 \mu$ s, consistent with regular-spike classifications (McCormick et al., 1985; Bruno and Simons, 2002).

Cells were reconstructed in three dimensions using a Neurolucida system with a $100 \times / 1.25 \mathrm{NA}$ oil-immersion objective. All tangential sections belonging to a cell were traced in their entirety and aligned using Neurolucida's Serial Section Manager before being spliced together. The barrel field was reconstructed at $4 \times$ on the basis of the $\mathrm{CO}$ staining. The barrel column identity of each layer $2 / 3$ pyramid was determined by following its main axon trunk into layer 4 . Control cells were located in the following columns: seven in D2, three in D3 and one in D4. The deprived group was distributed as follows: three in D1, seven in D2 and two in D3. To correct for possible variability in the plane of sectioning, the completed reconstruction was rotated 3-dimensionally so that its main axon trunk was located directly underneath the cell soma; this rotation did not change the statistical significance of any analyses.

We attempted to estimate synapse density by sampling segments of axon in the spared and deprived rows and manually counting varicosities along such segments. Manual identification of varicosities in this material using brightfield microscopy was highly subjective, preventing a reliable analysis of the true bouton density. Consequently, we do not report such data, which requires differently prepared tissue, other imaging techniques, and preferably objective counting methods.

Experimental blind. To prevent possible biases, the experiments, reconstructions, and data analysis were performed blind to the control/ deprived identity of the rats. The experimenter remained blind to identity by having someone else bring the animal to the lab on the day of the experiment. In the case of a control, this person would trim the A-C rows before bringing the rat to the lab. Each rat was assigned a number, which was then carried through all stages of cell analysis.

The blind was maintained during analyses using the following recoding method. Laboratory members who had trimmed all the whiskers off the day of the experiment reassigned control/deprived rats to groups A/B. By coin flip, they determined deprived would be group A. This mapping was kept secret until the analyses were completed. The data and the A/B identification (but not control/deprived identification) was given to a student who sorted the data by $\mathrm{A} / \mathrm{B}$, removed the rat numbers, labeled the sorted datasets groups $\mathrm{C}$ and $\mathrm{D}$, and gave the data to the data analyst. By coin flip, the student had determined that group A would be mapped to group $\mathrm{C}$ and kept this secret until the analyses were completed. The data analyst thus had two sorted groups $\mathrm{C}$ and $\mathrm{D}$ but did not know which corresponded to control/deprived. The blind was only broken after all analyses were completed.

Data analysis. Data were analyzed using NeuroExplorer (Microbright- 

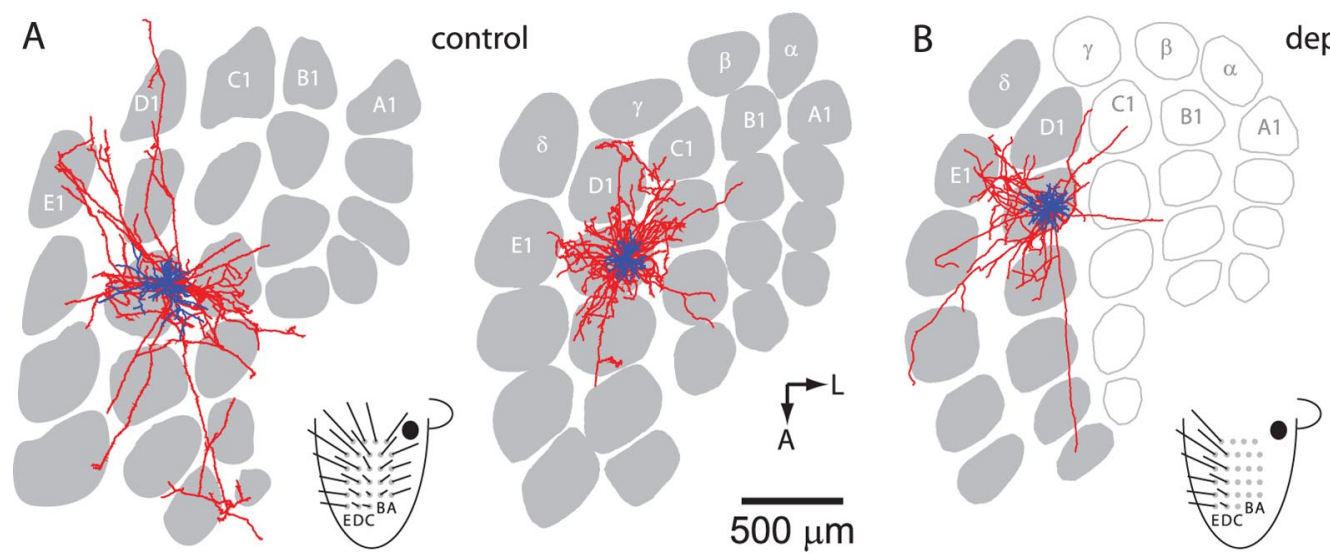

deprived
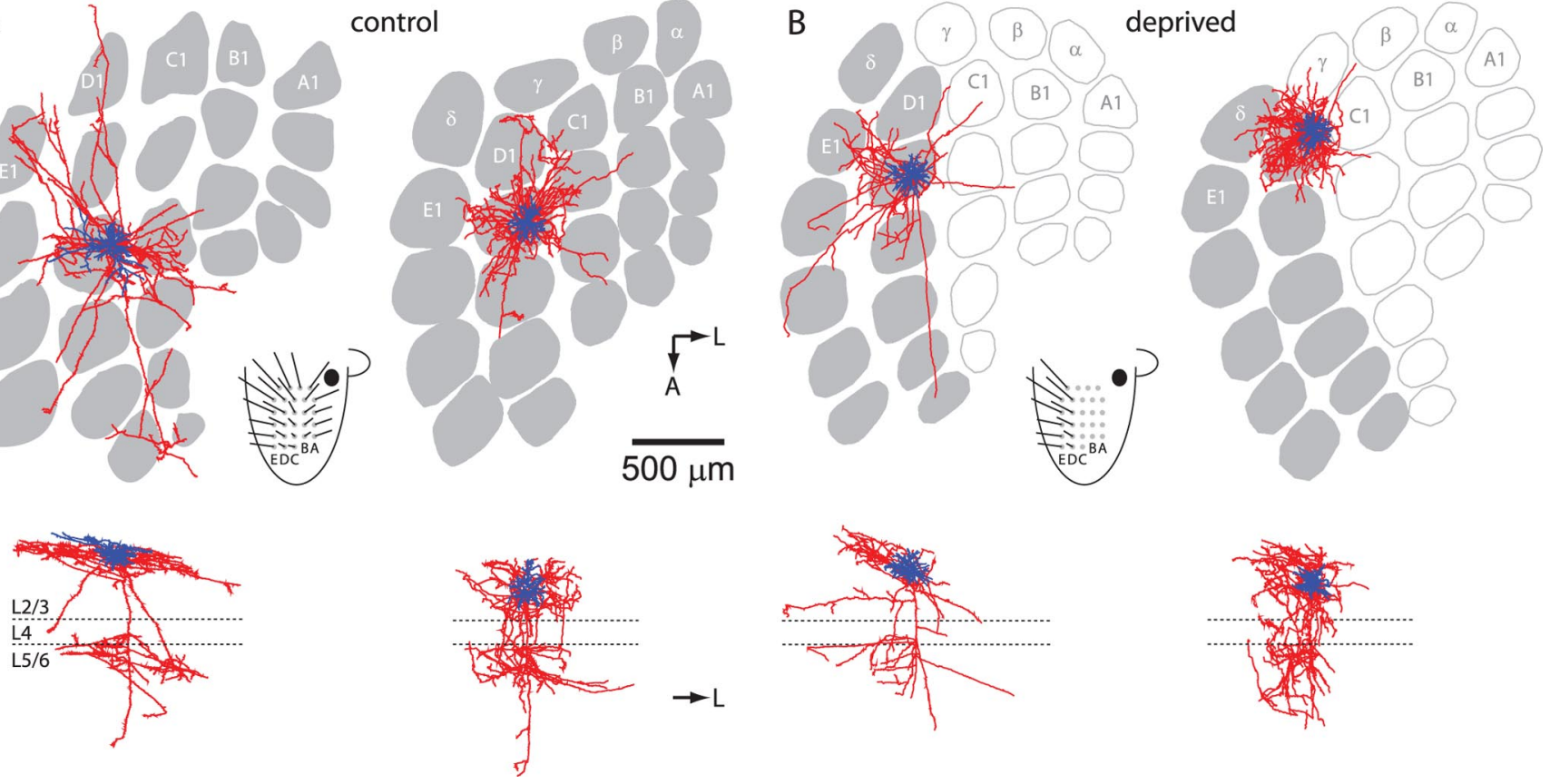

Figure 1. Example reconstructions of $L 2 / 3$ pyramidal neurons in normal and sensory-deprived rats. $A$, Axons (red), dendrites (blue), and barrel maps (gray) of two $L 2 / 3$ pyramidal neurons filled in two different control rats. Top, Tangential projections. Bottom, Corresponding coronal projections. A, Anterior; L, lateral. Inset, Schematic of rat face with whiskers intact. $\boldsymbol{B}$, Two cells filled in rats whose A-C whisker rows were deprived. Inset, schematic of trimmed rat face.

field), Excel, and custom-written Matlab routines. All statistical comparisons are nonparametric two-tailed (rank sum) tests unless otherwise noted. Means \pm SD are given throughout.

\section{Results}

From postnatal day 7, whisker rows A-C (including straddlers $\alpha-\gamma)$ of rats in the deprived group were trimmed to the level of the face each day whereas rows D-E were left intact (Fig. $1 B$, inset). Control littermates were sham trimmed (Fig. $1 A$, inset). After 12-17 d of trimming, animals were deeply anesthetized, the D row targeted by intrinsic signal imaging (supplemental Fig. S1, available at www.jneurosci.org as supplemental material) and/or extracellular recording, and single L2/3 pyramidal neurons in D-row barrel columns labeled in vivo by whole-cell or juxtasomal recording. Cells were histologically recovered and threedimensionally reconstructed using high-magnification brightfield microscopy ( $n=11$ control, 12 deprived). Experiments, reconstructions and analyses were performed blind to the control/deprived nature of the animals/cells.

The axons of filled L2/3 pyramids were often extensive, at times spanning millimeters of the barrel field (Fig. $1 A, B$, top row, red; supplemental Figs. S2-S5, available at www.jneurosci.org as supplemental material). Axons in the control group ranged from 14,274 to $59,570 \mu \mathrm{m}$ in total length (mean \pm SD: $38,565 \pm$ 13,$140 ;$ median 38,172). Total lengths of axons from the deprived group were significantly shorter, ranging from 7195 to $47,486 \mu \mathrm{m}$ (mean 25,093 $\pm 12,423$; median 24,145; $p=0.025$ ) (Fig. 2). Consistent with a reduction in total length, fewer branch points were observed in deprived axons (mean: $126 \pm 58$ per axon; median 117) than in controls (mean: $225 \pm 112$; median $217 ; p=$ 0.03 ) (Fig. 2). Total axonal length and number of branch points had a highly significant, moderately strong correlation $(r=0.76$, $p<0.0001$ ) (Fig. 2). The average distance between branch points, however, was similar for control and deprived groups $(p=0.78)$ as was the tortuosity of axonal segments between branch points $(p=0.88)$ (Table 1$)$.

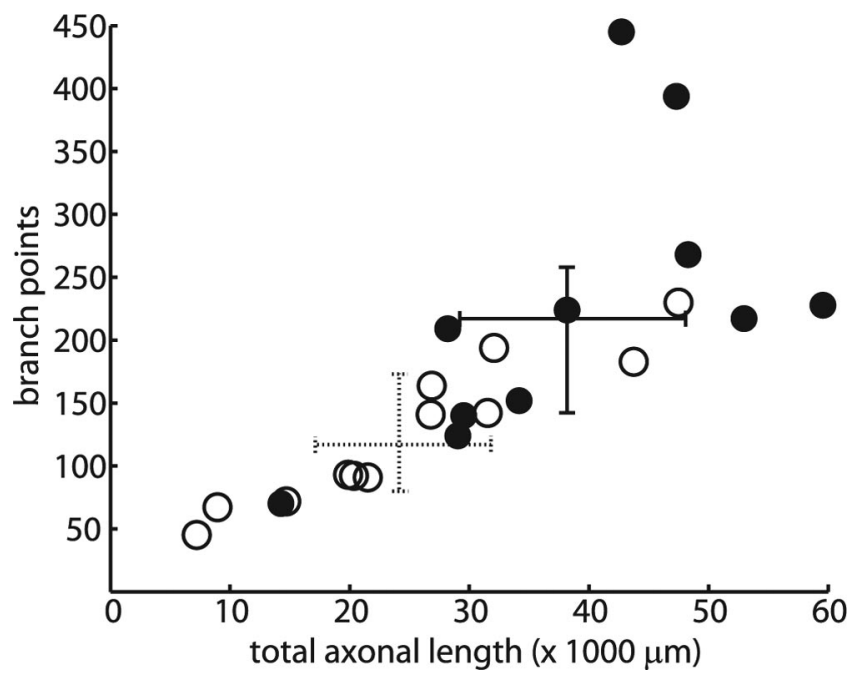

Figure 2. Deprivation decreases total axonal length and number of branch points. Filled circles, Control group axons; open circles, deprived group axons. Medians and 25-75\% percentiles are shown for control (solid crosshairs) and deprived (dashed crosshairs).

Axons of both control and deprived groups were distributed through the depth of the cortex in typical "butterfly" patterns (Fig. 1, bottom row) with on average $66 \%$ of the axon located in L2/3, $12 \%$ in layer 4 (L4), and $22 \%$ in layer $5 / 6$ (L5/6). Deprivation significantly reduced axonal length in L2/3 (from a median of 24,972 to 15,$423 ; p=0.025$ ). Total lengths in L4 and L5/6 were also decreased (from 3107 to 909 and from 11,441 to 4196 , respectively) but not significantly (Table 1). We observed no correlation of age or trim/sham duration with total axonal length or length within L2/3, L4, or L5/6 for either group or for the two groups combined ( $p$ values $>0.05)$. Thus, sensory deprivation during this period reduces overall length of axons emanating from L2/3 neurons 
Table 1. Comparison of axonal morphological measurements in control and deprived groups

\begin{tabular}{|c|c|c|c|c|c|}
\hline & $\begin{array}{l}\text { Control } \\
\text { (mean } \pm \text { SD) }\end{array}$ & $\begin{array}{l}\text { Deprived } \\
\text { (mean } \pm \text { SD) }\end{array}$ & Control median & Deprived median & $\begin{array}{l}p \text { value } \\
\text { (rank sum) }\end{array}$ \\
\hline Total axonal length $(\mu \mathrm{m})$ & $38,565 \pm 13,140$ & $25,093 \pm 12,423$ & 38,172 & 24,145 & 0.025 \\
\hline L2/3 axonal length ( $\mu \mathrm{m})$ & $23,119 \pm 6960$ & $15,571 \pm 6772$ & 24,972 & 15,423 & 0.025 \\
\hline L4 axonal length ( $\mu \mathrm{m})$ & $4617 \pm 3997$ & $3767 \pm 5145$ & 3107 & 909 & 0.21 \\
\hline L5/6 axonal length ( $\mu \mathrm{m})$ & $10,829 \pm 9806$ & $5755 \pm 5680$ & 11,441 & 4196 & 0.15 \\
\hline Total branch points & $225 \pm 112$ & $126 \pm 58$ & 217 & 117 & 0.03 \\
\hline$L 2 / 3$ length between branch points $(\mu \mathrm{m})$ & $95 \pm 25$ & $90 \pm 16$ & 95 & 86 & 0.78 \\
\hline L4 - 6 length between branch points $(\mu \mathrm{m})$ & $115 \pm 44$ & $86 \pm 34$ & 109 & 93 & 0.19 \\
\hline 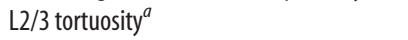 & $1.36 \pm 0.06$ & $1.35 \pm 0.12$ & 1.38 & 1.35 & 0.88 \\
\hline L4 $^{-6}$ tortuosity ${ }^{a}$ & $1.36 \pm 0.09$ & $1.45 \pm 0.16$ & 1.37 & 1.46 & 0.21 \\
\hline$L 2 / 3$ field span (row-wise, $\mu \mathrm{m}$ ) & $1726 \pm 741$ & $1317 \pm 461$ & 1821 & 1382 & 0.21 \\
\hline L2/3 field span (arc-wise, $\mu \mathrm{m}$ ) & $1236 \pm 432$ & $955 \pm 281$ & 1331 & 798 & 0.12 \\
\hline L4 - 6 field span (row-wise, $\mu \mathrm{m}$ ) & $1407 \pm 1027$ & $889 \pm 580$ & 1061 & 821 & 0.23 \\
\hline L4- 6 field span (arc-wise, $\mu \mathrm{m})$ & $1383 \pm 845$ & $746 \pm 380$ & 1139 & 927 & 0.053 \\
\hline
\end{tabular}

${ }^{a}$ For any given axonal segment, the ratio of process length between its branch points to the Cartesian distance between those branch points.

A

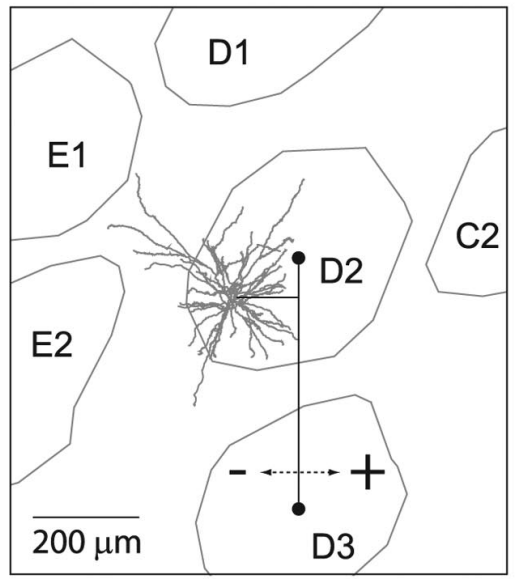

C

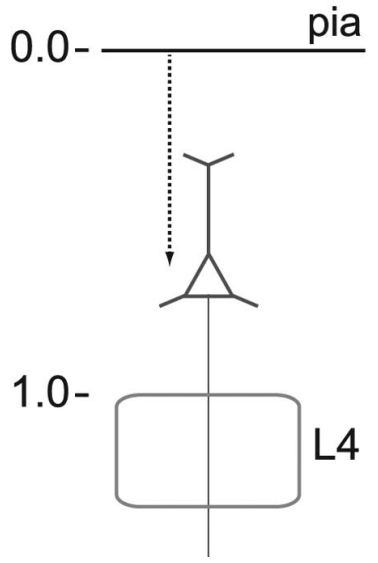

B

control

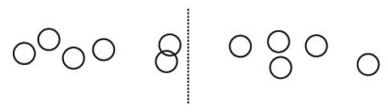

deprived

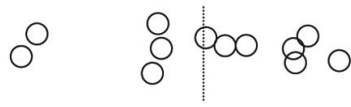

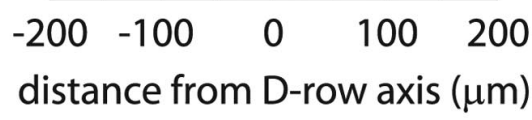

$\mathrm{D}$

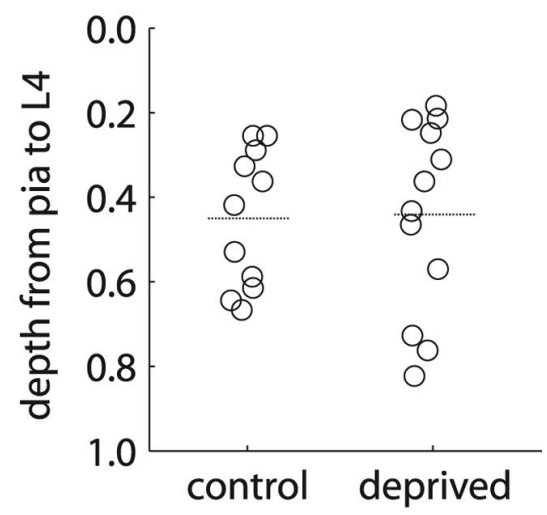

Figure 3. Neurons in control and deprived groups have similar spatial distributions. A, D-row axis (vertical line) was defined for each cell by calculating the centroids (black circles) of the barrel underlying the cell and the rostrally adjacent D-row barrel and determining the shortest distance from the soma to the axis (solid horizontal line). Cells on the E-side of the D-axis were defined to be negative, and cells on the C-side, positive. $\boldsymbol{B}$, Scatterplot of soma distances from the D-row axis for the control (top) and deprived (bottom) group. Data points have been vertically jittered for visualization. Dashed lines, Group means. C, Fractional depth of each soma was calculated as the ratio of its depth (from pia) to the depth of the top of L4, yielding a fraction from 0 to 1 . $D$, Jittered scatterplot of control and deprived groups' distributions of fractional depth. Dashed lines, Group means.

located in the spared D-row columns, with the largest effect on the horizontal connections within L2/3 itself.

\section{Spatial distributions of somata}

We checked that the two groups contained cells with similar spatial distributions. One of the groups could have been more closely located to the border between the spared and deprived areas, perhaps biasing its axonal arborization. Location of cell somata relative to their respective $D$-row axes were, therefore, measured in the tangential plane (Fig. 3A). Control and deprived somata were similarly distributed around the D-row axis (Fig. 3B), having means that were not significantly different (dashed lines, $t$ test $p=0.85$ ).

Recent functional studies in vitro suggest that horizontal connections within upper L2/3 may extend over longer distances and perhaps more columns than those in lower L2/3 (Bureau et al., 2006). We, therefore, further examined spatial distribution in this dimension. The depth of each soma was measured as the fractional distance from the pia to the top of $\mathrm{L} 4$ (Fig. 3C) to eliminate potential variability caused by fixation-induced shrinkage and deviations from the optimal tangential plane during slicing. Horizontal field spans of all axons in L2/3 were measured with respect to the $\mathrm{D}$-row axis and the orthogonal, nearly arc-oriented, axis. The relationship of soma depth and field span across the row, if any, was weak and barely trend-level $(r=0.33, p=0.11)$ (Fig. $4 A$, top). There was, however, a significant relationship of depth and field span across the $\operatorname{arc}(r=0.61, p=0.002)$ (Fig. $4 A$, bottom) with more superficial cells having larger field spans, a possible anatomical substrate of functional differences between upper and lower L2/3 (Bureau et al., 2006). There was no significant relationship of depth and total axonal length in L2/3 ( $p=0.36)$, suggesting that axons of upper $\mathrm{L} 2 / 3$ pyramids arborize over a wider territory but do not have greater length than axons originating from lower L2/3. Depth did not appear to influence field span in L4-6 (Fig. 4B). Separate regressions of control (filled circles) and deprived groups (open circles) did not change the statistical significance of these relationships. Thus, upper L2/3 pyramidal neu- 
rons generally have spatially more extensive supragranular axons than lower L2/3 pyramids do.

We, therefore, considered the possibility that control and deprived groups were accidentally sampled from markedly different depths. Depth distributions of control and deprived groups appeared, however, to be similar (Fig. 3D), having means (dashed lines) and variances that were not significantly different from each other $(t$ test, $p=0.75 ; F$ test for equal variances, $p=0.29$, respectively). Thus, control and deprived samples did not exhibit detectable differences in either horizontal locations or vertical depths of somata that could explain our finding that whisker trimming influences axonal arborization.

\section{Columnar organization of axonal arbors}

To investigate columnar organization, the spatial distributions of axons were quantified by a modified Sholl analysis (Sholl, 1953). Each axon was separated into a L $2 / 3$ and a L4-6 component by a virtual cut where the main trunk first descends into L4. An analysis grid of concentric circles subdivided every $45^{\circ}$ was placed over the center of the barrel underlying a neuron (Fig. $5 A$ ), and the three-dimensional length of axon occupying each bin measured for either the L2/3 or L4-6 component (Fig. 5B). The average L2/3 axon components of the control and deprived groups appear approximately symmetrically distributed with regard to the center of the barrel (Fig. $5 C$ ). The fraction of axonal arbor above the barrel did not differ for control (mean $21.9 \pm 11.6 \%$, median 21\%) and deprived (mean $24.8 \pm 11.5 \%$, median $24 \%$; $p=0.8$ ). The shorter overall length of the deprived group is also reflected in these density plots as well as in the plot of their difference (Fig. 5D), in which the reduction diffusely spreads in all directions. The L4-6 components exhibited similar spatial distributions with less axon observed in the deprived case (Fig. 5E,F). The differences between deprived and control groups have slightly asymmetric appearances for both the L2/3 and L4-6 components, but these are not statistically significant ( $p$ values $>0.05)$.

\section{Organization of individual axons}

The main effect of altered sensory experience, however, might be on individual L2/3 axonal arbors rather than the overall pattern of connectivity between cortical columns. To test this idea, we realigned the analysis grids to each cell's soma, rather than the center of the barrel underlying the soma, and measured axonal length in soma-centered coordinates. The average innervation map of the L2/3 component in controls is nearly symmetric with some bias toward the $\mathrm{C}$ row (Fig. $6 \mathrm{~A}$, left). In contrast, the axonal projections in the direction of the trimmed $\mathrm{C}$ rows are strikingly diminished in the deprived animals (Fig. 6A, right).

We statistically tested these differences by quantifying the axonal length in specific regions of interest (ROIs), $90^{\circ}$-wide and spanning 100-600 $\mu \mathrm{m}$ from the cell soma in the direction of the $\mathrm{C}$ or E row (Fig. 6A, gray). This interval was chosen because it contains the bulk of the axon, corresponds to the dimensions of 1-2 neighboring cortical columns, and excludes the axon's long
Layer 2/3

B
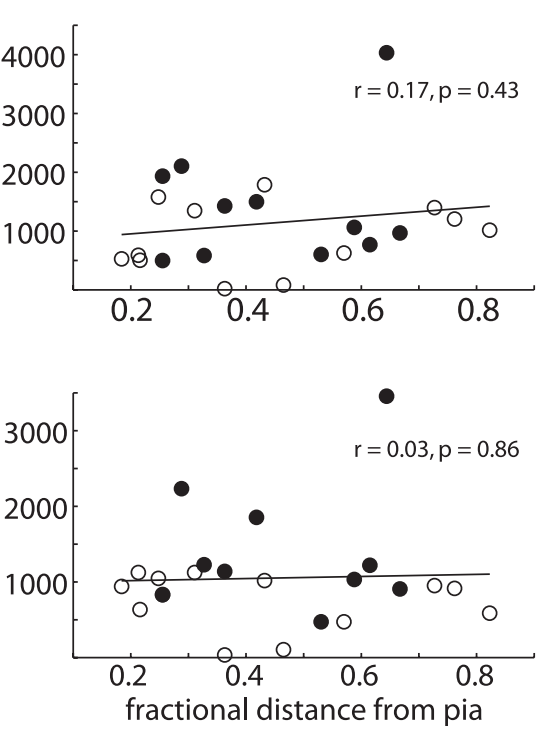

Figure 4. Analysis of axonal field span and cortical depth of soma. Filled circles, Control animals; open circles, deprived animals; do lower $L 2 / 3$ pyramids (bottom) but similar row-wise field spans (top). $\boldsymbol{B}$, Upper and lower $L 2 / 3$ pyramids did not have detectable when measured along the row (top) or arc (bottom).

radial trunk. On the C-row side, the ROI for the deprived group contained $68 \%$ less axon in L2/3 than the ROI for the controls (rank sum test, $p=0.001$ ) (Fig. $6 B$ ) (medians 3180 and $1031 \mu \mathrm{m}$ and means 4082 and $1328 \mu \mathrm{m}$ for control and deprived, respectively). A simple redistribution of axon from the $\mathrm{C}$ side to the $\mathrm{E}$ side cannot explain this result because the E-side ROI of the deprived group was not statistically different from that of controls $(p=0.17$ ) and was actually $22 \%$ less than controls (medians 4446 and $2177 \mu \mathrm{m}$ and means 4071 and $3163 \mu \mathrm{m}$ for control and deprived, respectively). Furthermore, comparison of control and deprived axonal lengths outside the C-side ROI barely reaches significance ( $p=0.05$; data not shown), suggesting that the highly significant decreases within the ROI account for much but not all of the overall length change.

A similar pattern of results was observed in the deeper layers: The C-side ROI was reduced by $51 \%$ for the L4-6 component $(p=0.04$ ) (Fig. 6C,D) (medians 1656 and $553 \mu \mathrm{m}$ and means 1829 and $900 \mu \mathrm{m}$ for control and deprived, respectively), and its E-side ROI by only $12 \%$, a statistically insignificant change ( $p=$ 0.64 ; medians 1156 and $938 \mu \mathrm{m}$ and means 1330 and $1173 \mu \mathrm{m}$ for control and deprived, respectively). Deprivation had no significant effect on the distance between branch points when only C-side or E-side branches were analyzed for either set of layers (supplemental Fig. S6, available at www.jneurosci.org as supplemental material) ( $p$ values $>0.1$ ).

The possibility that absolute differences in axonal density between control and deprived groups might be caused by an outlier, usually an unasymmetric cell, was examined by conducting a cell-by-cell analysis. The axonal lengths contained within the ROIs were plotted for each cell. Axonal branches in control animals were on average approximately symmetrically distributed both in L2/3 (Fig. $7 A ; p=0.9$, compare filled circle) and L4-6 (Fig. $7 B ; p=0.24$, compare filled circle). Deprivation appeared to reduce axonal length on the $\mathrm{E}$ side but not significantly $(p=0.17$ and 0.64 for L2/3 and L4-6, respectively), consistent with the 

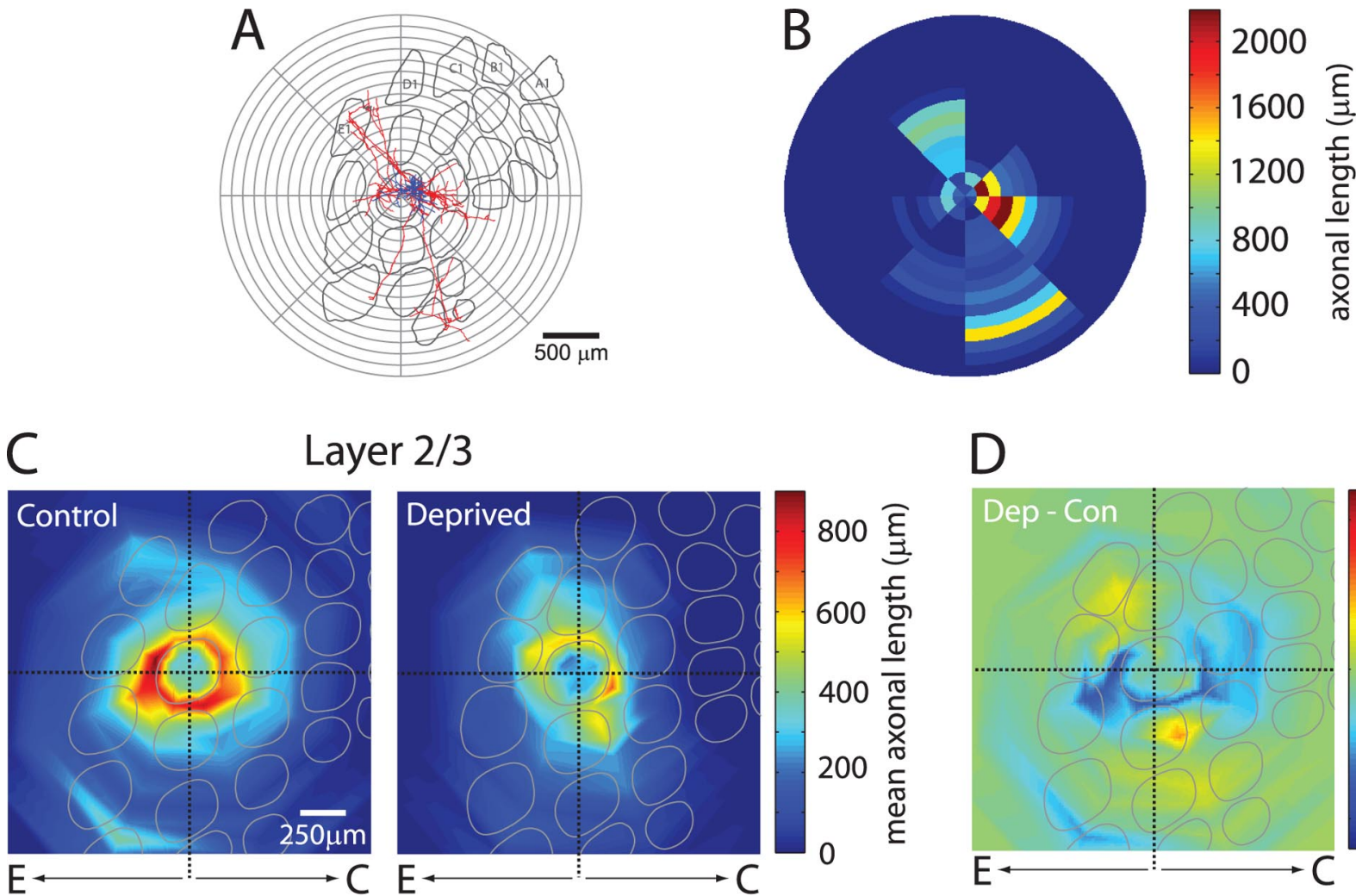

Layer $2 / 3$
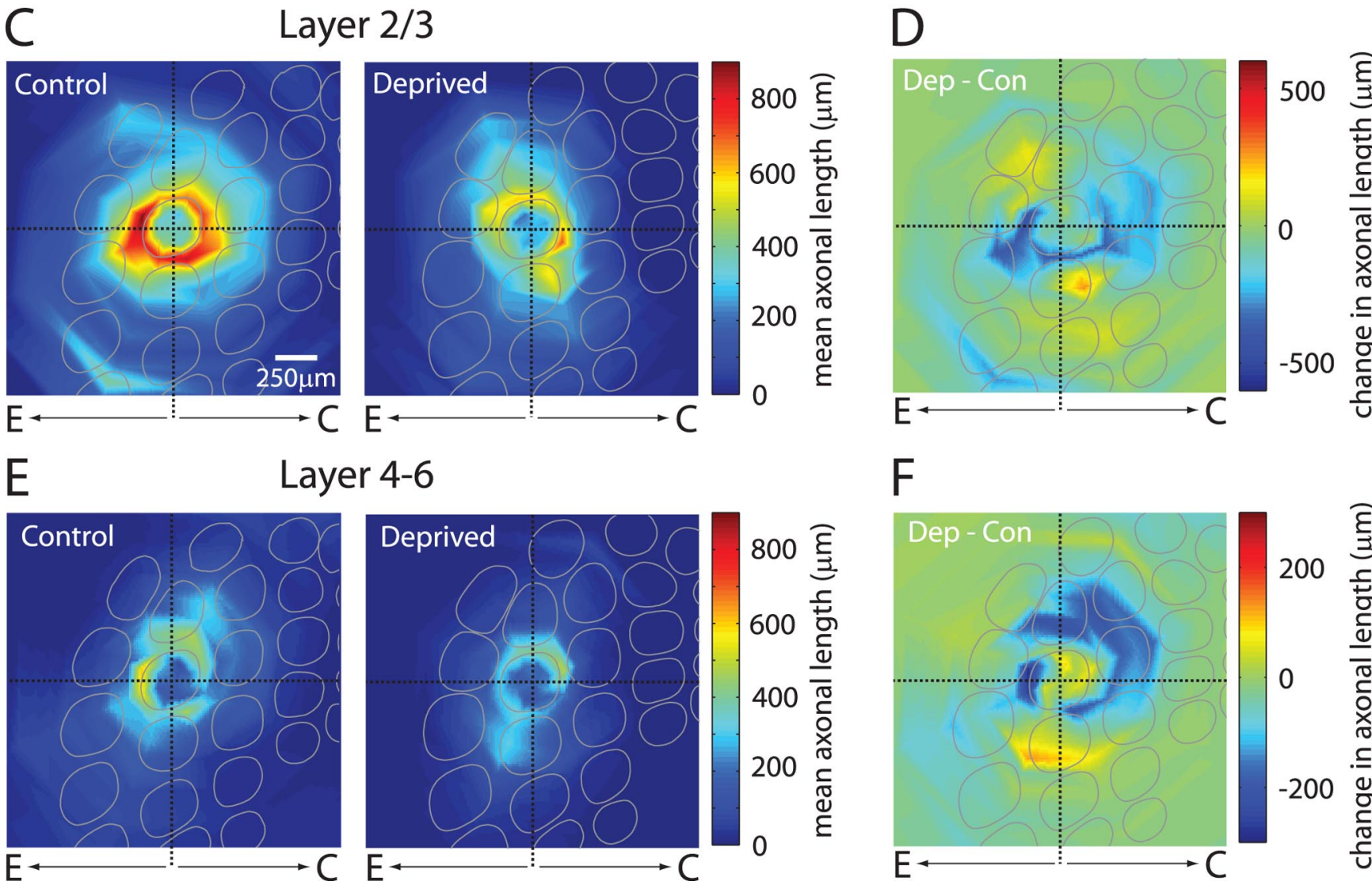

Figure 5. Barrel-aligned plots show that sensory deprivation decreases overall axonal innervation of the adjacent septum and neighboring cortical columns. $A$, Three-dimensional axonal lengths were quantified by placing a grid of concentric cylinders subdivided every $45^{\circ}$ on the barrel center and measuring the length of either the L2/3 or L4-6 axonal branches under each sector. Shown here are the L2/3 branches of the leftmost cell in Figure 1. B, Quantification of example shown in $A$. C, Spatial distribution of L2/3 axonal branch length in control (left) and deprived (right) rats. Each cell was quantified as in $\boldsymbol{B}$ and averaged by group. Smooth surfaces were estimated by interpolation of the spatially discretized averages. Average barrel maps were calculated for each group and superimposed over distributions. Downward, Rostral. Rightward, Lateral. Vertical line, D-row axis as defined in Figure 3. D, Subtraction of L2/3 control values from deprived values shown in C. $\boldsymbol{E}$, $\boldsymbol{F}$, Spatial distribution of $\mathrm{L} 4-6$ axonal branch length as in $\boldsymbol{C}, \boldsymbol{D}$.

diffuse overall reduced axonal lengths brought about by deprivation (Figs. 5D, 6B). More importantly, the localized decrease in axon on the trimmed $\mathrm{C}$ side, discussed above for Fig. 6, is apparent for multiple cells (Fig. 7): No one cell can account for the effects of deprivation.

Because the axons of L2 neurons tended to have larger field spans than those of L3 (Fig. 4), we investigated whether plasticity depended on soma depth. We further analyzed the ROI lengths in Fig. 7 by regressing it against fractional depth. Depth was unrelated to E-side or C-side ROI lengths ( $p$ values $>0.2$ ), consistent with our observation that depth is unrelated to total axonal length despite being correlated with field span. Furthermore, multiple regression of ROI lengths against fractional depth and trimming condition (control/deprived) revealed no interaction ( $p$ values $>0.2$ ). Total axonal length was similarly independent of depth and trimming condition ( $p$ values $>0.2$ ), suggesting that the plasticity we observed may not be limited to L2 or L3 but is rather a general $\mathrm{L} 2 / 3$ phenomenon.

Together, these analyses clearly demonstrate that sensory deprivation mainly reduces L2/3 axonal branches that extend in the direction of deprived columns and has little or no effect on arborization of branches in the direction of spared columns.

\section{Discussion}

How the nervous system wires itself has been a long-standing question. Most studies have focused on functional alterations 

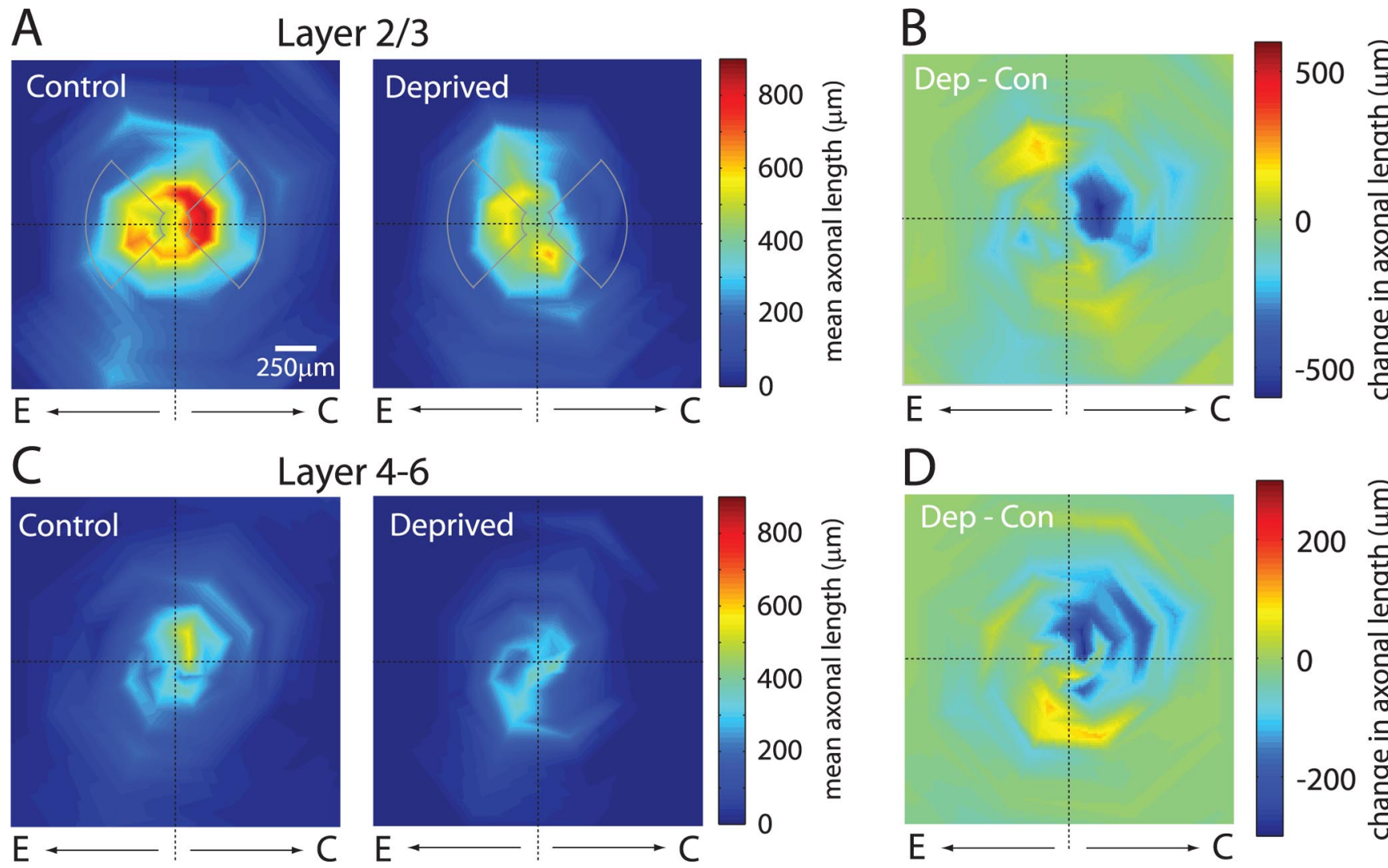

Figure 6. Soma-aligned plots show that deprivation decreases axonal innervation predominantly on a cell's deprived side. Quantifications were performed as in Figure 5 except that the analysis grid was centered on the soma rather than the barrel. $A$, Spatial distribution of L2/3 axonal branch length in control (left) and deprived (right) rats. Gray, Regions of interest compared between control and deprived. $\boldsymbol{B}$, Subtraction of $L 2 / 3$ control values from deprived values shown in $\boldsymbol{A}$. $\boldsymbol{C}, \boldsymbol{D}$, Analysis of spatial distribution of $L 4-6$ axonal branch length similar to that in $\boldsymbol{A}, \boldsymbol{B}$.

detected by electrodes or voltage/intrinsic signal imaging. Few have examined changes at the level of single-cell anatomy, and even fewer the effects on individual axons. We labeled single axons in layer $2 / 3$, long suspected to be a principal site of structural changes underlying functional reorganization (Buonomano and Merzenich, 1998; Fox, 2002; Feldman and Brecht, 2005). The axonal lengths we quantified are noteworthy: the arbor of a single $\mathrm{L} 2 / 3$ pyramidal cell may exceed $60 \mathrm{~mm}$ in barrel cortex alone. We discovered that neurons located superficially in L2/3, presumably L2, had more extensive axonal arbors than deeper neurons seemingly composing L3. This difference in connectivity between L2 and L3 may be the anatomical substrate of their previously described functional differences (Bureau et al., 2006). Both layers were, however, similarly plastic in terms of axonal length.

We found that the overall length of L2/3 axonal arbors emanating from spared territories decreases after whisker trimming. Functional reorganization of cortex, which has been described for many sensory modalities, is therefore not necessarily mediated solely by increases and decreases of the strength and dynamics of existing synaptic connections (Finnerty et al., 1999; Cheetham et al., 2007). Indeed, recent analyses of spine turnover suggest that new connections may be formed and existing ones pruned (Lendvai et al., 2000; Trachtenberg et al., 2002), a dendritic mechanism that reorganizes connectivity. Our data clearly show that corticocortical axons are also restructured and may complement dendritic modifications. Importantly, alteration of synaptic inputs and functional maps not only corresponds to local changes of axonal arbors but involves large-scale axonal structuring. Whether reduced arborization is the result of restriction of initial growth or pruning after normal growth requires further investigation, perhaps by reconstructing axons filled earlier within a similar deprivation period or by chronic in vivo imaging of single axons.

Surprisingly, mutual innervation of spared cortical columns (here the spared rows D and E) is not enhanced. Enhanced innervation might have been expected from a deprivation-induced increase in correlated activity between the spared columns (Fox, 2002; Feldman and Brecht, 2005). Spared columns could become more correlated by multiple mechanisms. L2/3 neurons in D row normally respond more robustly to C-row whisker deflections than neurons in $\mathrm{E}$ row, and this difference in surround receptive fields would have less consequence for activity after trimming. Also, spared whisker use may increase after trimming. Either mechanism of correlation might have triggered Hebbian-type plasticity between the two spared rows, but we found no evidence for such. Further experiments are needed to determine whether whisker pairing fails to increase correlated activity between spared columns or Hebbian plasticity has little role in axonal development.

Axons from spared areas did not invade deprived ones as has been described in adult cat visual cortex months after destructive retinal lesions (Darian-Smith and Gilbert, 1994). Additional studies are needed to determine whether the decreased innervation of deprived regions we observed is limited to development or results from our comparatively brief deprivation period or innoxious manipulation of the sensory periphery. Nevertheless, the observed axonal decrease, although spatially asymmetric and more pronounced in deprived than spared territory, does not perfectly match the underlying barrel borders that define the cortical columns. Intriguingly axonal change in $\mathrm{L} 2 / 3$ is better de- 

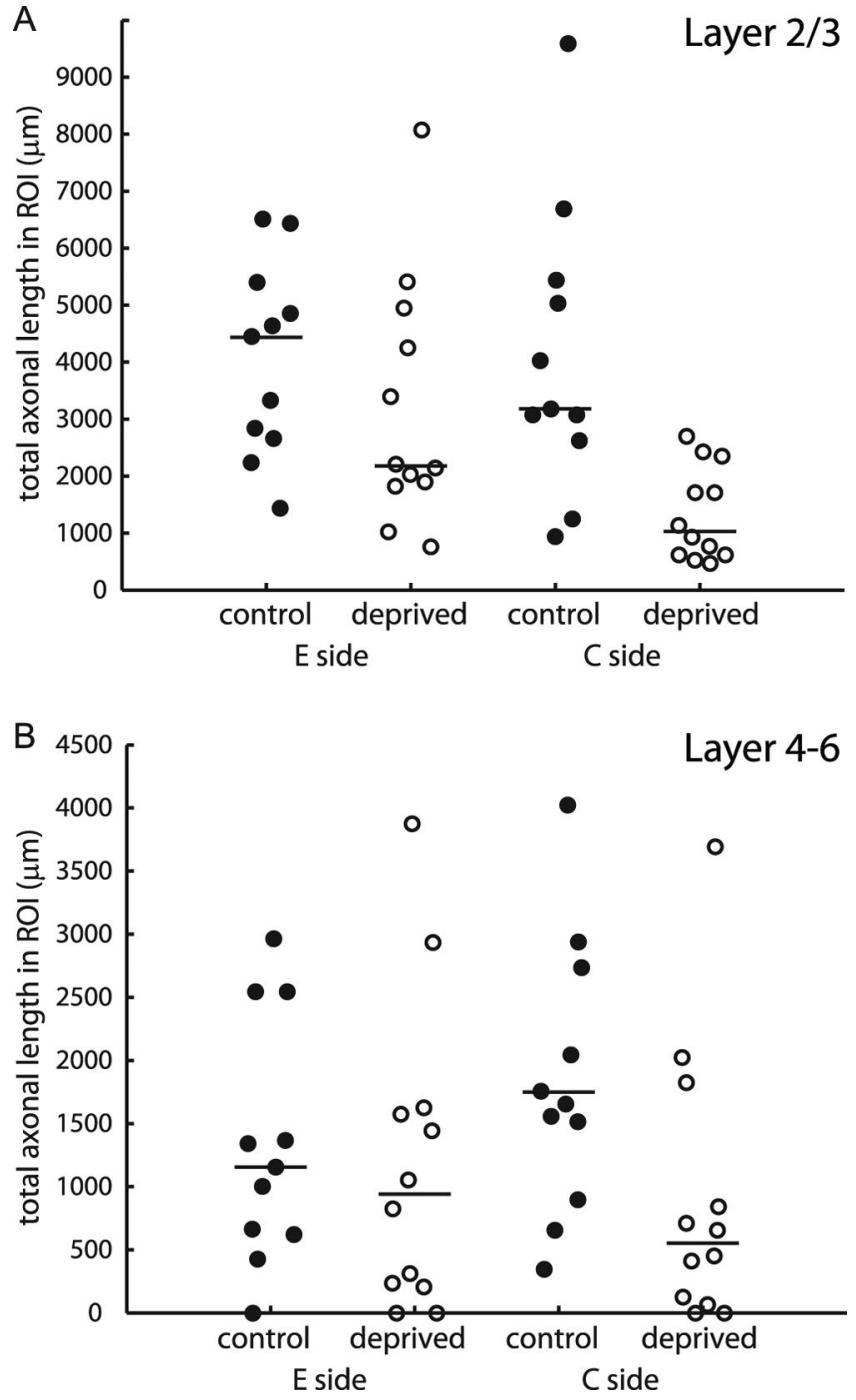

Figure 7. Cell-by-cell analysis of axonal length. $A$, The total length of the $L 2 / 3$ axonal branch contained within the ROIs defined by the gray lines in Fig. $6 \mathrm{~A}$ is plotted for each cell. Symbols are horizontally jittered for visualization. Filled, Control animals; open, deprived animals; lines, medians. $\boldsymbol{B}$, Same as $\boldsymbol{A}$, but for the $L 4-6$ axonal branch.

scribed by a reduction of those axonal branches arborizing in the direction of deprived territories, independent of whether or not the branch is actually in a spared/deprived area. Thus, at least in development, sensory experience impacts axonal arborization not just between but within cortical columns.

Why should anatomical plasticity be more apparent when we consider the spatial locations of axonal branches relative to their cell bodies (Fig. 6) rather than cortical columns (Fig. 5)? The answer may lie in the continuous rather than discrete organization of L2/3 even in barrel cortex, well known for its discretely organized L4 (Woolsey and Van der Loos, 1970). Anatomically, barrel column boundaries are often crossed not just by axons but also dendrites of L2/3 pyramids (supplemental Figs. S2, S3, available at www.jneurosci.org as supplemental material), similar to dendrites of L5B pyramids (Ito, 1992) and even many L4 cells (Elston et al., 1997). Functionally, the adjacent whiskers evoking the most rapid and strong action potential responses from a L2/3 neuron are those corresponding to the neighboring barrel columns closest to the soma (Armstrong-James et al., 1992; Glazewski and Fox, 1996), and the magnitude of this surround response is distance-dependent (Kerr et al., 2007; Sato et al., 2007).
Trimming whisker rows A-C is therefore likely to immediately alter the gradient of activity not just across the barrel field but also within individual columns comprising the spared D row. Axonal branches on different sides of the soma lie within zones having different degrees of responsiveness to adjacent whiskers, making the soma-centered effect the most noticeable change. Our findings suggest that L2/3 is anatomically and functionally organized in a continuous rather than discrete manner, contradicting extreme notions of columnar organization in cortex.

A blurred, weaker column-centered effect should be detectable with a single-cell dataset many times larger than ours, but such an experiment is impractical. Bulk loading of hundreds to thousands of neurons (Löwel and Singer, 1992; Trachtenberg and Stryker, 2001; Broser et al., 2008) is well suited to testing such a columnar effect. Indeed, using the same trimming paradigm, Broser et al. (2008) showed that axonal populations in L2/3 are more asymmetric in deprived animals. Population studies cannot, however, measure how individual axons change. Similarly, because bulk labeling fills a variable number of cells, the absolute amount of axonal change remains unknown and necessitates the use of relative measures. The high density of labeled material within the first few hundred micrometers of an injection site further precludes examination of intracolumnar organization. Using orders of magnitude fewer cells, we observed a robust branchspecific decrease extending over 50-350 $\mu \mathrm{m}$ (Fig. 6), a region which includes both intra- and intercolumnar modifications.

Axonal plasticity was not limited to supragranular layers but also involved the $\mathrm{L} 2 / 3$ projection to infragranular $\mathrm{L} 5 / 6$, a pathway whose plasticity has not been previously demonstrated. Using bulk tracers Broser et al. (2008) detected no major effect of trimming on the columnar organization of axons in infragranular layers, nor did we (Fig. $5 E, F$ ). Trimming reduced, however, arborization of axonal branches in L4-6 on the deprived side of the soma (Fig. 6C,D), albeit less than in L2/3. Deep layers develop earlier than superficial layers (Rice et al., 1985) and are thereby more mature and presumably less malleable during trimming in our paradigm. However, L4-6 also differ substantially from L2/3 in spontaneous and evoked activity (Simons, 1978; ArmstrongJames and Fox, 1987; de Kock et al., 2007). The activity of L2/3 pyramids may therefore correlate more strongly with other neurons in L2/3, rather than L4-6, of neighboring columns. Correlation differences may limit induction of spike-timing dependent plasticity and consequently both the stabilization of synaptic inputs to deep layers and the magnitude of changes later observable there. Consistent with such a role of correlated/uncorrelated activity patterns, blockade of spontaneous activity in organotypic slice cultures causes axons of L6 pyramidal neurons to lose their normal preference for L4 over L5 and instead arborize equally in both layers (Dantzker and Callaway, 1998). Alternatively, decreased activity of deprived areas may simply alter the levels of some trophic factor released to initially attract and/or stabilize developing axons of L2/3 pyramids. Whichever mechanism is at play, rewiring of the L2/3-L5/6 pathway is a likely substrate of receptive field plasticity in infragranular layers observed after whisker trimming (Diamond et al., 1994).

Various deprivation paradigms can over days/weeks change L2/3 receptive fields, measured by postsynaptic potentials and action potentials (Fox, 2002; Bureau et al., 2006; Wallace and Sakmann, 2008). Despite experience-induced changes of L4-L2/3 connection strength in vitro (Shepherd and Svoboda, 2005), single-cell filling suggests little plasticity of L4 axonal projections to L2/3 after whisker plucking (Bender et al., 2003). In the visual system, reconstruction of selected bulk-filled geniculocortical ax- 
ons demonstrated decreased arborization within topographically corresponding columns after monocular deprivation (Antonini and Stryker, 1993a), but intercolumnar axons have not been similarly measured. Many studies of cortical plasticity have implicated changes in synaptic strength and dynamics as well as spine motility and morphology (Finnerty et al., 1999; Feldman and Brecht, 2005; Majewska et al., 2006). Our reconstructions of how individual axons innervate a large cortical area indicate that sensory deprivation also limits the arborization of specific axonal branches in a spared area, a conclusion that would have been difficult to infer from physiological techniques such as paired recording or local extracellular stimulation. Assuming relatively constant bouton spacing, these substantial axonal length changes, up to $68 \%$ reductions, are likely to result in decreases in the number of excitatory synapses. Future experiments, perhaps using electron microscopy and/or immunohistological labeling of presynaptic proteins, are needed to examine whether synapse density undergoes compensatory increases or enhancing decreases. Synapse density may simply be unaffected given that the large axonal changes described here already can account for the alteration of sensory representations after deprivation (Wallace and Sakmann, 2008).

\section{References}

Antonini A, Stryker MP (1993a) Rapid remodeling of axonal arbors in the visual cortex. Science 260:1819-1821.

Antonini A, Stryker MP (1993b) Development of individual geniculocortical arbors in cat striate cortex and effects of binocular impulse blockade. J Neurosci 13:3549-3573.

Antonini A, Stryker MP (1996) Plasticity of geniculocortical afferents following brief or prolonged monocular occlusion in the cat. J Comp Neurol 369:64-82.

Antonini A, Stryker MP (1998) Effect of sensory disuse on geniculate afferents to cat visual cortex. Vis Neurosci 15:401-409.

Antonini A, Gillespie DC, Crair MC, Stryker MP (1998) Morphology of single geniculocortical afferents and functional recovery of the visual cortex after reverse monocular deprivation in the kitten. J Neurosci 18:9896-9909.

Armstrong-James M, Fox K (1987) Spatiotemporal convergence and divergence in the rat S1 "barrel" cortex. J Comp Neurol 263:265-281.

Armstrong-James M, Fox K, Das-Gupta A (1992) Flow of excitation within rat barrel cortex on striking a single vibrissa. J Neurophysiol 68:1345-1358.

Bender KJ, Rangel J, Feldman DE (2003) Development of columnar topography in the excitatory layer 4 to layer $2 / 3$ projection in rat barrel cortex. J Neurosci 23:8759-8770.

Bourne J, Harris KM (2007) Do thin spines learn to be mushroom spines that remember? Curr Opin Neurobiol 17:381-386.

Broser P, Grinevich V, Osten P, Sakmann B, Wallace DJ (2008) Critical period plasticity of axonal arbors of layer $2 / 3$ pyramidal neurons in rat somatosensory cortex: layer-specific reduction of projections into deprived cortical columns. Cereb Cortex 18:1588-1603.

Bruno RM, Simons DJ (2002) Feedforward mechanisms of excitatory and inhibitory cortical receptive fields. J Neurosci 22:10966-10975.

Buonomano DV, Merzenich MM (1998) Cortical plasticity: from synapses to maps. Annu Rev Neurosci 21:149-186.

Bureau I, von Saint Paul F, Svoboda K (2006) Interdigitated paralemniscal and lemniscal pathways in the mouse barrel cortex. PLoS Biol 4:e382.

Calford MB (2002) Dynamic representational plasticity in sensory cortex. Neuroscience 111:709-738.

Callaway EM, Katz LC (1991) Effects of binocular deprivation on the development of clustered horizontal connections in cat striate cortex. Proc Natl Acad Sci U S A 88:745-749.

Cheetham CE, Hammond MS, Edwards CE, Finnerty GT (2007) Sensory experience alters cortical connectivity and synaptic function site specifically. J Neurosci 27:3456-3465.

Chklovskii DB, Mel BW, Svoboda K (2004) Cortical rewiring and information storage. Nature 431:782-788.

Dantzker JL, Callaway EM (1998) The development of local, layer-specific visual cortical axons in the absence of extrinsic influences and intrinsic activity. J Neurosci 18:4145-4154.

Darian-Smith C, Gilbert CD (1994) Axonal sprouting accompanies functional reorganization in adult cat striate cortex. Nature 368:737-740.

de Kock CP, Bruno RM, Spors H, Sakmann B (2007) Layer- and cell-typespecific suprathreshold stimulus representation in rat primary somatosensory cortex. J Physiol 581:139-154.

Diamond ME, Huang W, Ebner FF (1994) Laminar comparison of somatosensory cortical plasticity. Science 265:1885-1888.

Elston GN, DeFelipe J (2002) Spine distribution in cortical pyramidal cells: a common organizational principle across species. Prog Brain Res 136:109-133.

Elston GN, Pow DV, Calford MB (1997) Neuronal composition and morphology in layer IV of two vibrissal barrel subfields of rat cortex. Cereb Cortex 7:422-431.

Feldman DE, Brecht M (2005) Map plasticity in somatosensory cortex. Science 310:810-815.

Finnerty GT, Roberts LS, Connors BW (1999) Sensory experience modifies the short-term dynamics of neocortical synapses. Nature 400:367-371.

Fox K (2002) Anatomical pathways and molecular mechanisms for plasticity in the barrel cortex. Neuroscience 111:799-814.

Fox K, Wong RO (2005) A comparison of experience-dependent plasticity in the visual and somatosensory systems. Neuron 48:465-477.

Glazewski S, Fox K (1996) Time course of experience-dependent synaptic potentiation and depression in barrel cortex of adolescent rats. J Neurophysiol 75:1714-1729.

Grinvald A, Lieke E, Frostig RD, Gilbert CD, Wiesel TN (1986) Functional architecture of cortex revealed by optical imaging of intrinsic signals. Nature 324:361-364.

Horikawa K, Armstrong WE (1988) A versatile means of intracellular labeling: injection of biocytin and its detection with avidin conjugates. J Neurosci Methods 25:1-11.

Ito M (1992) Simultaneous visualization of cortical barrels and horseradish peroxidase-injected layer $5 \mathrm{~b}$ vibrissa neurones in the rat. J Physiol 454:247-265.

Kaas JH (2002) Sensory loss and cortical reorganization in mature primates. Prog Brain Res 138:167-176.

Kasai H, Matsuzaki M, Noguchi J, Yasumatsu N, Nakahara H (2003) Structure-stability-function relationships of dendritic spines. Trends Neurosci 26:360-368.

Kerr JN, de Kock CP, Greenberg DS, Bruno RM, Sakmann B, Helmchen F (2007) Spatial organization of neuronal population responses in layer 2/3 of rat barrel cortex. J Neurosci 27:13316-13328.

Kleinfeld D, Delaney KR (1996) Distributed representation of vibrissa movement in the upper layers of somatosensory cortex revealed with voltage-sensitive dyes. J Comp Neurol 375:89-108.

Larsen DD, Callaway EM (2006) Development of layer-specific axonal arborizations in mouse primary somatosensory cortex. J Comp Neurol 494:398-414.

Lendvai B, Stern EA, Chen B, Svoboda K (2000) Experience-dependent plasticity of dendritic spines in the developing rat barrel cortex in vivo. Nature 404:876-881.

Löwel S, Singer W (1992) Selection of intrinsic horizontal connections in the visual cortex by correlated neuronal activity. Science 255:209-212.

Majewska AK, Newton JR, Sur M (2006) Remodeling of synaptic structure in sensory cortical areas in vivo. J Neurosci 26:3021-3029.

Margrie TW, Brecht M, Sakmann B (2002) In vivo, low-resistance, wholecell recordings from neurons in the anaesthetized and awake mammalian brain. Pflugers Arch 444:491-498.

McCormick DA, Connors BW, Lighthall JW, Prince DA (1985) Comparative electrophysiology of pyramidal and sparsely spiny stellate neurons of the neocortex. J Neurophysiol 54:782-806.

Pinault D (1996) A novel single-cell staining procedure performed in vivo under electrophysiological control: morpho-functional features of juxtacellularly labeled thalamic cells and other central neurons with biocytin or neurobiotin. J Neurosci Methods 65:113-136.

Rice FL, Gomez C, Barstow C, Burnet A, Sands P (1985) A comparative analysis of the development of the primary somatosensory cortex: interspecies similarities during barrel and laminar development. J Comp Neurol 236:477-495.

Sato TR, Gray NW, Mainen ZF, Svoboda K (2007) The functional microarchitecture of the mouse barrel cortex. PLoS Biol 5:e189. 
Shepherd GM, Svoboda K (2005) Laminar and columnar organization of ascending excitatory projections to layer $2 / 3$ pyramidal neurons in rat barrel cortex. J Neurosci 25:5670-5679.

Sheth BR, Moore CI, Sur M (1998) Temporal modulation of spatial borders in rat barrel cortex. J Neurophysiol 79:464-470.

Sholl DA (1953) Dendritic organization in the neurons of the visual and motor cortices of the cat. J Anat 87:387-406.

Simons DJ (1978) Response properties of vibrissa units in rat SI somatosensory neocortex. J Neurophysiol 41:798-820.

Trachtenberg JT, Stryker MP (2001) Rapid anatomical plasticity of horizontal connections in the developing visual cortex. J Neurosci 21:3476-3482.

Trachtenberg JT, Chen BE, Knott GW, Feng G, Sanes JR, Welker E, Svoboda
K (2002) Long-term in vivo imaging of experience-dependent synaptic plasticity in adult cortex. Nature 420:788-794.

Wallace DJ, Sakmann B (2008) Plasticity of representational maps in somatosensory cortex observed by in vivo voltage-sensitive dye imaging. Cereb Cortex 18:1361-1373.

Woolsey TA, Van der Loos H (1970) The structural organization of layer IV in the somatosensory region (SI) of mouse cerebral cortex. The description of a cortical field composed of discrete cytoarchitectonic units. Brain Res 17:205-242.

Xu HT, Pan F, Yang G, Gan WB (2007) Choice of cranial window type for in vivo imaging affects dendritic spine turnover in the cortex. Nat Neurosci 10:549-551. 